\title{
LE RELIEF DU SUD-OUEST DE L'ANGOLA SELON MARIANO FEIO
}

Un important mémoire, daté de 1981, mais seulement diffusé en 1984, rend compte des recherches géomorphologiques réalisées par MARIANO FEIO en Angola, de 1958 à 1966.

Mariano FEIo, O Relevo do Sudoeste de Angola. Estudo de Geomorfologia, Memórias da Junta de Investigações Científicas do Ultramar, 2." série, 67, Lisboa, 1981, 326 p., 58 fig., 19 phot., résumés portugais, français et anglais.

La région présentée, qui s'étend sur environ $200000 \mathrm{~km}^{2}$, comporte fondamentalement trois unités: le vaste plateau intérieur ou Plateau Principal, doucement incliné vers le Sud-Est et que draine, à l'Ouest, le réseau exoréique du Cunene; le grand escarpement qui limite à l'Ouest le plateau; la Surface Intermédiaire et le bassin sédimentaire qui frange le littoral atlantique.

Il est vivement conseillé, avant d'entreprendre la lecture du corps de l'ouvrage, de lire attentivement un des trois substantiels résumés qui l'accompagnent. L'essentiel des faits d'observation significatifs, des propositions d'interprétation et des discussions de problèmes y apparaissent avec une grande netteté. Il sera ensuite beaucoup plus facile au lecteur de tirer parti d'un texte dense, de présentation généralement austère et qui donne volontairement le premier pas à la description minutieuse des faits choisis par l'auteur en fonction de la lumière qu'ils apportent à la compréhension de l'organisation régionale des formes et de sa probable évolution. 
L'ouvrage commence par une mise au point sur le cadre naturel et sur la documentation existante (essentiellement, la carte topographique au 1: 100000 , avec courbes de niveau espacées de $50 \mathrm{~m}$ et une couverture de photographies aériennes dont l'échelle varie de 1: 28000 à 1: 40000 ). On notera en particulier les cartes climatiques, élaborées avec des données mises à jour jusqu'en 1972, la carte pétrographique (fig. 6) qui regroupe les roches selon leur comportement géomorphologique et le commode résumé des publications antérieures sur l'évolution du relief de la région: les travaux de nombreux chercheurs allemands (P. VAGELER, O. JESSEN, W. BEETZ, E. ObSt et K. KAYSer, H. A BEL) et d'un Hollandais, F. J. FABER.

L'étude géomorphologique présentée par MARIANO FEIO se caractérise par une séparation volontairement très nette entre une minutieuse description, clairement et sèchement ordonnée, de tous les éléments importants du paysage, toujours présentés dans leurs rapports avec la structure géologique, description longuement mise à la disposition du lecteur (p. 61 à 263), avant que l'auteur ne se décide à lui offrir une proposition d'évolution du relief, discutée et schématisée en une dizaine de pages (p. 277 à 286). C'est dire qu'il s'agit d'un ouvrage austère, solide, fait pour durer et pour fournir aux futurs chercheurs le meilleur de 23 mois de travail de terrain et de nombreuses années d'une réflexion ample, prudente et rigoureuse, impitoyable pour ses propres faiblesses, mais cependant capable d'embrasser en une vision grandiose les temps géologiques et les espaces démesurés du socle africain.

L'étude du relief, dont la synthèse est donnée par la carte géomorphologique (fig. 17), débute par la présentation de l'élément central de son organisation, dans la région où celle-ci est la plus nette, c'est à dire le complexe escarpement en escalier qui relie le plateau intérieur aux basses régions atlantiques (p. 61-109). Le Plateau Principal, qui s'étend du Nord au Sud de l'Angola, présente une remarquable uniformité. Dans la région étudiée, l'orientation des rivières qui s'y encaissent souligne son inclinaison d'ensemble vers le Sud. Le plateau de Humpata-Bimbe, qui atteint $2300 \mathrm{~m}$ d'altitude, domine le Plateau Principal par de nets rebords d'érosion et est lui-même dénivelé en trois parties au long d'antiques flexures. L'auteur considère qu'il s'agit d'une surface aplanie en fonction du niveau de base local constitué par ses rebords quartzitiques et qu'elle ne semble pas correspondre à une ancienne surface d'érosion cyclique de grande extension. A l'Ouest, le plateau est brusquement limité par le grandiosé escarpement de la Chela, qui atteint une dénivellation de $1500 \mathrm{~m}$ là où il est couronné par des quartzites, alors que les formes du rebord occidental du Plateau Principal s'atténuent dans les régions granitiques, en gardant toujours cependant la même importance décisive dans le paysage. Au pied de l'escarpement s'étend la Surface dite Intermédiaire, limitée à l'Ouest par une autre surface, dite de la Flexure, qui plonge sous la couverture sédimentaire littorale, comme le montre la figure 19, ici reproduite (fig. 1). La Surface Intermédiaire est une vaste pédiplaine imparfaite, encore encombrée de reliefs résiduels, surtout dans sa partie orientale.

Le chapitre suivant traite de la géologie du bassin sédimentaire littoral (p. 111148). Quatre séries chronologiques sont distinguées, décrites et utilisées pour établir la succession des événements tectoniques et géomorphologiques. L'auteur s'appuie en 
bonne partie sur loeuvre de G. SoARES DE CARvalHo (1961), tout en discutant certaines de ses interprétations, ainsi que celles de M. TELLES ANTUNES (1964). La succession des couches crétacées permet de mettre en évidence un important mouvement tectonique d'âge Albien-Aptien, suivi d'une transgression limitée au Cénomanien-Turonien, puis d'une autre plus accentuée au Maestrichien, alors que la région se trouvait à nouveau approximativement aplanie. La série de la base du Tertiaire, dite Eocène, présente des "accidents de sédimentation" que les géologues avaient considérés comme des discordances d'origine tectonique, mais que M. FEIO pense n'être que les caractéristiques normales d'une sédimentation de plaine littorale. Après une longue lacune de sédimentation correspondant à l'Oligocène, apparait une formation littorale à conglomérat de base, datée du Miocène par ses fossiles, puis un autre conglomérat, probablement Plio-Quaternaire.

Sont ensuite considérées les formes du plateau oriental qui s'abaisse lentement en direction du bassin endoréique du Cuanhama (p. 149-169). Sa surface porte, çà et là, les restes d'une croûte de roches silicifiées d'âge incertain, qui disparait souvent sous les sables de la formation dite du Kalahari. Deux cycles d'érosion emboités se reconnaissent dans la vallée du Caculovar qui entaille le plateau et débouche, vers $1120 \mathrm{~m}$ d'altitude, dans la vaste plaine de sédimentation endoréique du bassin subsident de l'Etocha Pan. Les sédiments d'origine fluviale s'y entassent sur quelque $300 \mathrm{~m}$ et leurs couches supérieures incorporent des outils paléolithiques. La morphologie de détail est héritée de l'époque où la surface de remblaiement à chenaux anastomosés était encore active, avant que sa partie occidentale n'ait été capturée par le Cunene.

Les caractéristiques et l'évolution de ce dernier fleuve, long de $1220 \mathrm{~km}$, sont longuement traitées dans le chapitre suivant (p. 171-225). Son régime hydrologique contrasté est caractérisé par les données disponibles (5 années, de 1963/64 à 1967/68, en 6 stations), puis son cours est décrit d'amont en aval. Jusqu'après Roçadas, vers 17. 24' $\mathrm{S}$, le profil longitudinal accompagne les déformations du plateau, d'abord sur le socle ancien où la vallée est régulièrement encaissée d'une centaine de mètres, ce qui parait correspondre à l'épaisseur des roches altérées, ensuite sur les sédiments du bassin du Cuanhama. Près de Roçadas, la vallée présente une nette dissymétrie que

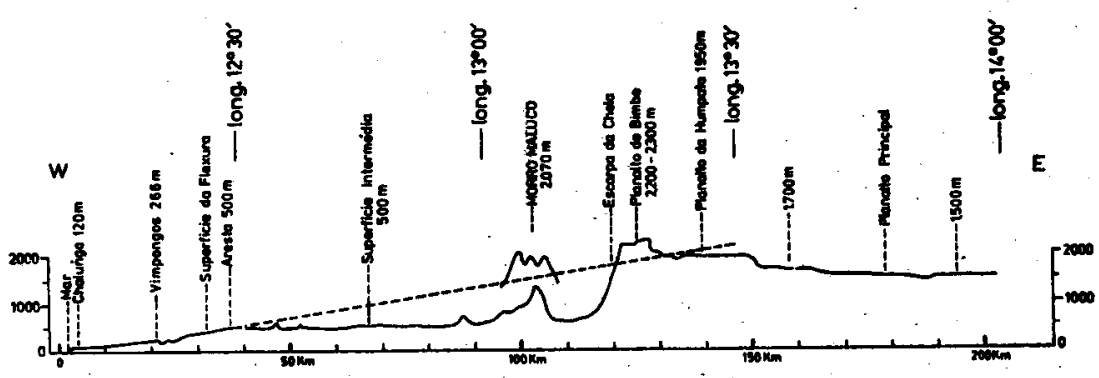

Fig. 1-Profil, au long du parallèle $15^{\circ} \mathrm{S}$, depuis l'océan jusqu'au Plateau Principal. Hauteurs $\times 10$. (Figure 19 de l'ouvrage). 
M. FEIO est tenté d'attribuer à l'action de la force de Coriolis. Ne pourrait-on pas évoquer une possible influence néotectonique, analogue à celle qui marque diverses vallées du Ribatejo? D'autant plus que l'auteur admet sans difficulté, comme on va le voir, d'autres déformations tectoniques quaternaires. La "capture" du Cunene, suggérée par le changement de direction et l'accentuation brutale de l'inclinaison du profil, est ensuite discutée, après une soigneuse caractérisation des accidents principaux qui affectent son cours à l'aval de la chute de Ruacana. Une série de profils longitudinaux et transversaux et de cartes de détail illustrent les principaux aspects décrits. Il est un peu dommage qu'une carte de localisation d'ensemble n'aide pas la gymnastique de lecture complexe, indispensable pour donner à chaque détail sa place et son poids dans l'ensemble du cours. M. FEIO discute d'abord l'hypothèse de BEETZ (1950), selon laquelle l'ancien Cunene gagnait déjà l'Atlantique au long du cours actuel du Curoca. Selon lui, le Curoca a failli au contraire capturer lateralement le Cunene inférieur, mais le processus a été interrompu par un événement de tout autre ampleur. Selon l'interprétation proposée (fig. 42), le Cunene supérieur se serait autrefois divisé en bras divergeants dont certains atteignaient l'Etocha Pan, tandis qu'un autre s'avançait vers le Sud-Ouest, puis vers l'Ouest, où des restes de la couverture sédimentaire s'observent jusque vers $13 .^{\circ} \mathrm{E}$ (fig. 28), fossilisant encore en partie la surface du Chitado que l'auteur pense être emboitée dans le Plateau Principal, de façon analogue aux formes reconnues plus à l'Est, dans la vallée du Caculovar. L'inclinaison vers l'Ouest de la surface du Chitado fait penser qu'elle a été déformée tectoniquement et que cette déformation progressive serait responsable de la déviation du Cunene vers l'extrémité occidentale du bassin intérieur. Le détournement vers l'océan résulterait lui-même soit d'un déversement, soit d'une capture par le pré-Cunene inférieur. Le détail de la reconstitution demeure forcément très vague, mais on voit qu'elle implique nécessairement un rejeu tectonique quaternaire du bourrelet occidental africain.

Le reste du livre comporte une série de courts chapitres où M. FEIO résume successivement ses observations sur le désert sableux méridional et les grands cordons littoraux atlantiques, sur la région de transition; d'interprétation malaisée, qui prolonge au Sud le plateau de Humpata-Bimbe et où se détache le vaste plateau structural du Curoca, formé de grès et de dolérite. Il présente ensuite les formes structurales principales de la région, en particulier les vallées de fracture et les crêtes correspondant aux dikes doléritiques. Il regroupe en un court chapire les formes de modelé les plus typiques des trois grands domaines climatiques de l'espace étudié (climat tropical humide à saison sèche, climat semi-aride et climat aride). Enfin est présenté de façon succinte un schéma d'évolution du relief qui rassemble les conclusions partielles des chapitres antérieures. Si l'on admet que la Surface de la Flexure se reliait au Plateau Principal lors de l'achèvement de celui-ci à la fin du Crétacé, on est amené à penser que leur déformation serait antérieure au conglomérat de la base du Miocène. Ce schéma est comparé aux synthèses proposées antérieurement par JESSEN, OBST et KAISER et L. KING; référence est alors faite à une Surface Infé- 
rieure, développée au Nord de $14 .^{\circ} \mathrm{S}$ au long du littoral angolais, mais qui n'est pas visible dans la région étudiẻe.

S. DAVEAU 